\title{
Karl Grandjot und der Dedekindsche Rekursionssatz
}

\author{
Christoph Lamm
}

Über den 1922 bei Edmund Landau promovierten Mathematiker Karl Grandjot wissen wir viel weniger als über die übrigen Landau-Schüler dieser Zeit, wie z.B. Gustav Doetsch, Alexander Ostrowski, Carl Ludwig Siegel und Erich Kamke. Dafür gibt es zwei Gründe: Nachdem Grandjot ab 1929 in Chile tätig war, publizierte er nicht mehr in internationalen Fachzeitschriften und seine Haupttätigkeit war die Modernisierung der mathematischen und physikalischen Ausbildung in Chile. Der andere Grund ist, dass die Würdigung seiner Tätigkeit in der spanischsprachigen Literatur stattfand.

So ist im Jahr 2004 der biografische Artikel [15] von Claudio und Flavio Gutiérrez über den - in Chile Carlos Grandjot genannten - Göttinger Mathematiker erschienen. Um den Inhalt zugänglicher zu machen, wurde er auf meine Anregung kürzlich von Karin Erxmeyer übersetzt [16]. Der vorliegende Artikel möchte durch eine knappe Zusammenfassung Lust auf die Lektüre der Übersetzung machen - oder bei ausreichenden Spanischkenntnissen auf die des Originals - und ergänzendes Material liefern.

Bekannt ist Karl Grandjot heute wohl nur wegen seiner Erwähnung im Vorwort von Edmund Landaus „Grundlagen der Analysis“ (1930), [26]. Dieses Buch hat eine historische Bedeutung, da in ihm zum ersten Mal der Aufbau des Zahlensystems von den natürlichen Zahlen zu den rationalen, reellen und schließlich komplexen Zahlen durchgeführt worden ist. Es wird außerdem wegen seiner beiden prägnant geschriebenen Vorworte (,Vorwort für den Lernenden“ und „Vorwort für den Kenner") oft zitiert.' Landau berichtet dort, dass Grandjot ihn am Ende des Sommersemesters 1927 auf eine Lücke bei der Definition der Addition der natürlichen Zahlen in seinem Skript aufmerksam gemacht hatte. Diese Episode veranlasste Landau, sein Buch „Grundlagen der Analysis“ zu schreiben.

Vor Landau hatte bereits Richard Dedekind in seinen berühmten Schriften „Stetigkeit und irrationale Zahlen“ (1872) und „Was sind und was sollen die Zahlen?“” (1888) den Aufbau des Zahlensystems streng begründet. $\mathrm{Da}$ er jedoch die natürlichen Zahlen mengentheoretisch

126. Satz der Definition durch Induktion. Ist eine beliebige (ähnliche oder unähnliche) Abbildung $\theta$ eines Systems $\Omega$ in sich selbst und außerdem ein bestimmtes Element $\omega$ in $\boldsymbol{\Omega}$ gegeben, so gibt es eine und nur eine Abbildung $\psi$ der Zahlenreihe $N$, welche den Bedingungen

I. $\psi(N) 3 \Omega$,

II. $\psi(1)=\omega$,

III. $\psi\left(n^{\prime}\right)=\theta \psi(n)$ genügt, wo $n$ jede Zahl bedeutet.

Abbildung I. Der Dedekindsche Rekursionssatz, I888 [5] konstruierte, während Landau von den auf Dedekinds Werk beruhenden Axiomen ausging (,Dedekind-PeanoAxiome"), konnte es zu der genannten Lücke im Aufbau kommen. Der hier wesentliche Satz ist der Dedekindsche Rekursionssatz (siehe Abbildung I) und wir wollen „Grandjots Einwand“ und Dedekinds 100. Todestag zum Anlass nehmen, die Geschichte des Dedekindschen Rekursionssatzes zusammen mit den biografischen Ergänzungen zu Karl Grandjot darzustellen.

Daher besteht dieser Artikel aus folgenden Abschnitten: Biografisches zu Karl Grandjot, Dedekinds Rekursionssatz bis zum Erscheinen der "Grundlagen der Analysis“ (1930) und als dritter Abschnitt die neuen Beweise des Rekursionssatzes von Paul Lorenzen und László Kalmár von 1939/40 und deren Wirkung bis ungefähr 1990.

Der „Wunderstudent":

In sechs Semestern zur Promotion

Karl Grandjots Vorfahren waren Hugenotten, die vermutlich aus Donzère/Rhône nach Hessen gekommen waren. Geboren am 23. August 1900 in Frankenberg/Hessen besuchte Grandjot in Kassel die Oberrealschule bis 1919 und begann das Studium zum Sommersemester 1919 in Göttingen. Nach nur 6 Semestern promovierte er dort im Alter von 21 Jahren bei Edmund Landau über ein Thema aus der analytischen Zahlentheorie. Wie es zu der sehr kurzen Studiendauer kam, entnehmen wir Landaus Dissertations-Gutachten ${ }^{2}$ vom 31. Januar 1922:

Im Buch von Hardy und Riesz über Dirichletsche Reihen wird Bohr ein Beispiel einer Reihe $\sum_{n=1}^{\infty} \frac{a_{n}}{n^{s}}$ zugeschrieben, für welche die Konvergenzabszisse $\alpha=0$, die absolute Konvergenzabszisse $\beta=1$ ist, und außerdem bei allen $\delta>0, \epsilon>0$ für $\sigma>\delta$ die Funktion $O\left(|t|^{\epsilon}\right)$ ist (d. h. die Lindelöfsche $\mu$ Funktion $\mu(\sigma)=0$ für $\sigma>0$ ist). Ein solches Beispiel steht aber gar nicht bei Bohr, und ich hatte Herrn Grandjot die Aufgabe gestellt, eines zu bilden. Zur größten Überraschung (auch meines Freunds Hardy) entdeckte G. (durch Anwendung einer zu anderen Zwecken von Carlson kürzlich publizierten Beweismethode), dass es gar kein solches Beispiel gibt, nämlich dass für jede Reihe $\sum_{n=1}^{\infty} \frac{a_{n}}{n^{s}}$ mit $\alpha=0$, $\beta=1$ sogar $\mu(\sigma) \geq \frac{1}{2}$ ist. [...]

Diese neuen Resultate reichen zweifellos zu einer Dissertation. Dass sie so rasch zu beweisen gehn, kann ihren Wert nicht vermindern, also die Annahme des Man[uskripts] als Diss. nicht verhindern. 
Weil allerdings Carlsons (G. aus meiner Vorlesung rasch bekanntgewordenes) Paradigma die schlimmsten Steine bereits aus dem Weg geräumt hatte, schlage ich nur Zulassung zur mündlichen Prüfung mit dem Prädikat gut (III) vor.

Bei der Dissertation fällt außerdem ihr geringer Umfang auf: Sie besteht inklusive Titelblatt aus nur 12 Seiten. Nach der Promotion wurde Grandjot Landaus Assistent und war so an der Vorbereitung der Vorlesungen beteiligt. Von 1921 bis 1924 war dies ein 6-semestriger Zahlentheorie-Zyklus, der 1927 in drei Bänden als „Vorlesungen über Zahlentheorie“ von Landau veröffentlicht wurde. Im Artikel von C. und F. Gutiérrez ist erwähnt, dass Grandjot mindestens ein Drittel des Werks selbst geschrieben habe. Für die damalige Zeit war es jedoch nicht ungewöhnlich, dass ein Assistent nicht als Koautor aufgeführt war. ${ }^{3}$ Diese Arbeit fällt in die Zeit seiner Habilitation, die 1925 erfolgte - mit gerade noch 24 Jahren.

Die folgenden Ausschnitte aus Landaus HabilitationsGutachten vom 7. Juli 1925 zeigen, dass Landau seinen Schüler weiterhin sehr schätzte. Sie enthalten auch interessante Bemerkungen zum heute noch ungelösten Dirichletschen Teilerproblem und der Zusammenarbeit mit dem Zahlentheoretiker Harald Bohr, dem Bruder des Physikers Niels Bohr.

Ich begrüsse es mit besonderer Freude, dass nach längerer Pause wieder einmal einer meiner Schüler so weit ist, dass ich seine Zulassung als Privatdozent befürworten kann. Herr Grandjot ist aus der Generation, die vom Sommersemester 1919 ab meinen Kursus vom Anfang an hörte; unter den vielen guten Schülern, die ich damals hatte, fiel er mir sofort als der beste auf. Er hat seither meine gute Prognose aufs glänzendste bestätigt. Schon vor seinem siebentem Semester promovierte er mit einem selbstgewählten Thema; über seine Dissertation habe ich seinerzeit der Fakultät ausführlich berichtet.

Über seine inzwischen gedruckten Arbeiten ist zu sagen:

I) Sein neuer Beweis der Irreduzibilität der Kreisteilungsgleichung unterbietet noch an Kürze seine Dissertation. Und das ist das Wertvolle daran. Auch unter Benutzung des Dirichletschen Satzes war kein ähnlich einfacher Beweis jenes Satzes bekannt. Dass auch Mertens auf analogem Wege war, ist nur schmeichelhaft für den Verfasser. [...]

Ich komme nun zur Habilitationsschrift, die bei weitem die wertvollste seiner bisherigen Untersuchungen ist. Sie besteht aus zwei wesentlich getrennten Teilen.

Um beim Bericht über den I. Teil von hinten anzufangen: Ein ganz spezieller Fall seiner neuen allgemeinen Sätze setzt in Evidenz, dass die berühmte absolute Konstante des Dirichletschen Teilerproblems $\geq \frac{1}{4}$ ist, was Hardy und ich vor 10 Jahren unabhängig fanden, aber nur durch Benutzung spezieller Eigenschaften der Riemannschen $\zeta$-Funktion. [...]

Ich und Bohr, der gerade hier ist, sehen nach dem Studium seiner Arbeit viel klarer durch die ganze Theorie, mit der wir beide uns seit Jahrzehnten abmühen.

Im 2. Teil stellt er den Anschluss zwischen meinen Untersuchungen über Wurzeln Dirichletscher Reihen und den Wennbergschen her und verallgemeinert beide sehr.

Die Arbeit ist äußerst wertvoll.

Vor allem aber ist der ganze Mann ein ernster Gelehrter mit tiefen und vielseitigen Kenntnissen, scharfer Kritik, rascher Auffassungsgabe; er ist auch sehr erfahren im Unterricht und im Verkehr mit den Studierenden, trägt sehr gut vor, ist produktiv begabt und wird in jeder Hinsicht ein nützliches Mitglied unseres Lehrkörpers sein.

Da er seit über 3 Jahren mein einziger Assistent ist, habe ich ihn in allen Beziehungen erprobt und weiss gewiss, dass er auch in seiner neuen Thätigkeit (bei der er die alte nicht aufgeben soll) uns viel leisten wird.

Seine Vorlesungstätigkeit begann Grandjot im Sommersemester 1926 mit einer 4-stündigen Vorlesung ,Projektive Geometrie“ und einer I-stündigen Vorlesung „Mathematische Unterhaltungen und Spiele“. Über dieses heute etwas überraschend erscheinende Thema hatte auch Landau bereits 1912 und 1919 gelesen. Im Wintersemester 1926/27 folgte eine 3-stündige Vorlesung „Einführung in die Gruppentheorie“. Da Landau das Wintersemester 1927/28 in Jerusalem verbringen wollte, übernahm Grandjot die im Sommer beginnenden Anfängervorlesungen, nämlich „Differential- und Integralrechnung“ und "Grundlagen der Analysis“ und überraschte Landau am Ende des Sommersemesters damit, eine Lücke in dessen Skript gefunden zu haben, was Thema des nächsten Abschnitts ist.

Nur skizzenhaft sei hier Grandjots Biografie ab 1928 dargestellt. In diesem Jahr nahm er am Internationalen Mathematiker-Kongress in Bologna teil und hielt sich gefördert durch ein Rockefeller-Stipendium ${ }^{4}$ - in Paris bei Gaston Julia auf. Das Stipendium brach er im Frühjahr $1929 \mathrm{ab}$, obwohl noch ein Aufenthalt in Cambridge bei John Littlewood geplant war, und reiste nach Chile. Schwerpunkt seiner Tätigkeit dort war die Ingenieur- und Lehrerausbildung. Mit der aus Göttingen mitgebrachten „,modernen Mathematik“ hatte er keinen Erfolg. So veröffentlichte er 1940 seine „Algebra Abstracta“, eine Einführung in die Algebra auf Spanisch, welche ungefähr den Inhalt der ersten vier Kapitel von Bartel van der Waerdens „Moderne Algebra“ (1930), [42] abdeckt. ${ }^{5}$ Ein Textbeispiel über euklidische Ringe ist in Abbildung 2 zu sehen. Die Zeit war in Chile aber offenbar noch nicht reif für die abstrakte Algebra und die Einführung blieb unbeachtet. ${ }^{6}$ 
\$34. Por otra parte son algo frecuentes los anillos en que todo ideal es ideal principal; en ellos, por supuesto, ta noción de ideal es poco menos que superflua. Un tipo importante de tales anillos sencillos se caracteriza por la existencia de un "algoritmo de Euclides". Recordemos qué se entiende por esto. En Eo, si tratamos de encontrar el máximo común divisor de $a, b(\neq 0)$ dividiremos $a$ por $b$; obtendremos un resto $c$ de valor absoluto inferior al valor absoluto de $b$; continuaremos con $b$, $c$, etc.; después de un número finito de repetinuaremos con $b, c$, etc.; después de un numero finito de repeticiones, obtendremos, en el momento en que la division resulta exacta, como último divisor al M. C. D. de $a, b$. El mismo procedimiento puede aplicarse en el caso de $C[x]$, en que $C$ es un cuerpo; en vez de bajarse el "valor absoluto" se bajará aquí el "grado" de los restos sucesivos. Si se designa el grado de un polinomio $\neq 0$ como su "valor absoluto" se pued abarcar, entre otros, los dos casos mencionados en la definición siguiente:

DEF. 31: UN DOMINIO DE INTEGRIDAD $E$ SE LLAMA EUCLIDEANO CUANDO SE PUEDE HACER COEUCLIDEANO CUANDO SE PUEDE HACER CO RRESPONDER A TODO a $\# 0$ DE E UN NU MERO ENTERO SOLUTO" TAL QUE PARA TODO PAR DE ELEMENTOS $a, b$ DIFERENTES DE CERO SE TIENE 1) $|a b| \geqq|a|$ : 2) $b=a q+r$ DONDE $r=0$ ó $|r|<|a|$.

TEOR. 22: EN UN DOMINIO DE INTEGRIDAD EUCLIDEANO TODO IDEAL ES IDEAL PRINCIPAL.

Demostración: Si el ideal $A$ consta sólo del cero es $A=$ (0). Si no, sea $a$ un elemento $\neq 0$ de $A$ cuyo valor absoluto (que es un número entero no negativo) sea mínimo. Siendo $b$ otro elemento $\mp 0$ de $A$ tenemos $b=a q+r ;$ como $b \varepsilon A$ $a \varepsilon A$, $a q \varepsilon A, r=b-a q \varepsilon A$ no puede ser $|r|<|a|$, sino será $r=0$. Esto significa $b=a q$, luego $A=(a)$.

§35. Dirijamos nuestra atención, en estas circunstancias, al estudio de los ideales principales. De $a=b$ se sigue (a) $=(b)$; pero, ¿qué se puede inferir de $(a)=(b)$ ? Se ve que deben regir, siendo $e, f$ elementos de $E$, las relaciones $a=e b, b=f a$; luego ef $=u$. El elemento $c$ tiene, pues, recíproco $e^{-1}$ en $E$; un tal elemento se llama una unidad de $E$ (ino se confunda con "el" elemento uno!).

Abbildung 2. Euklidische Ringe in der Algebra Abstracta, 1940

Einen Einblick in die Atmosphäre in Göttingen gibt der Bericht von Freunden und Kollegen, dass Grandjot „gestand“, in Göttingen des starken Konkurrenzdrucks und der Rivalität überdrüssig gewesen zu sein [17]. Er wünschte sich ein ruhigeres Leben, was er dann in Chile bei geologischen und botanischen Exkursionen zusammen mit seiner Frau Gertrud, einer Biologin, verwirklichen konnte. Der Göttinger Botaniker Gerhard Wagenitz erhielt über einen Kontakt zu dem deutschchilenischen Botaniker Otto Zöllner Informationen über Grandjot und seine Frau, und es gibt in seinem 1988 erschienenen Buch [44] über Göttinger Biologen einen Eintrag zu Karl Grandjot. Einige Pflanzennamen enthalten übrigens den Namen Grandjot, z. B. „Myrceugenella grandjotii“"

\section{Die Geschichte der induktiven Definition} bis 1930

Wir wenden uns nun der Geschichte des Dedekindschen Rekursionssatzes zu.

\section{Begriff}

Als Erstes ist zu bemerken, dass Dedekind einen anderen Begriff verwendete. Eine Definition der Art $f\left(n^{\prime}\right)=$ $\varphi(f(n))$ mit Startwert $f(1)=$ a nannte er nicht ,,rekursive Definition“, sondern, wie in Abbildung I gezeigt, ,Definition durch Induktion“ (hier bezeichnet, wie üblich, $n^{\prime}$ den Nachfolger von $n$ ). Da dadurch leicht Verwechslungen mit dem „Beweis durch vollständige Induktion“ passieren konnten, wurde später der Begriff ,,rekursive Definition" verwendet, so zum Beispiel von Paul Bernays im Jahr 1935 [3]. In den 30 Jahren bis 1965 kamen beide Varianten vor. Seither benutzt man fast nur noch die Bezeichnung ,rekursive Definition“ und der Satz über die Existenz der so definierten Funktionen heißt daher „Rekursionssatz".

\section{Beispiele}

Einfache Beispiele für rekursive Definitionen sind die des Summenzeichens, $\sum_{i=1}^{1} a_{i}:=a_{1}, \sum_{i=1}^{n^{\prime}} a_{i}:=\sum_{i=1}^{n} a_{i}+$ $a_{n^{\prime}}$ und jene der Potenzierung, $a^{1}:=a, a^{n^{\prime}}:=a^{n} \cdot a$. Die hier auftretenden Operationen + und $\cdot$ werden für natürliche Zahlen wiederum rekursiv definiert: $a+1:=a^{\prime}$; $a+b^{\prime}:=(a+b)^{\prime}$ und $a \cdot 1:=a ; a \cdot b^{\prime}:=a \cdot b+a$. Da die zu definierende Operation jedoch auf beiden Seiten der jeweils zweiten Gleichung auftritt, muss man beweisen, dass die gewünschten Funktionen tatsächlich existieren.

An folgendem Beispiel sehen wir, dass die Existenz nicht selbstverständlich ist. Wir wollen die Eigenschaften „gerade“ und „ungerade“ definieren und setzen „I ist ungerade“, , $n^{\prime}$ ist gerade/ungerade $\Leftrightarrow n$ ist ungerade/gerade“" 7 Auf der Menge der natürlichen Zahlen funktioniert diese rekursive Definition - auch ohne Kenntnis der Addition, Multiplikation und Teilbarkeit. Wendet man sie aber auf einen verwandten Zahlbereich wie $\mathbb{Z} / 7 \cdot \mathbb{Z}$ mit $\bar{\sigma}^{\prime}=\overline{0}$ an, dann ergibt sich ein Widerspruch.

\section{Dedekind und Peano}

Dedekind arbeitete über mehrere Jahrzehnte an einem strengen Aufbau des Zahlensystems und beeinflusste mit Georg Cantor und Ernst Zermelo auch die Entwicklung der Mengenlehre. ${ }^{8}$ Nachdem Dedekinds „Was sind und was sollen die Zahlen?" I888 erschienen war, übernahm Giuseppe Peano die grundlegenden Eigenschaften als Axiome und veröffentlichte sie in seinen Schriften ab 1889. Aus diesem Grund wurden sie häufig nach Peano benannt, obwohl sie von Dedekind stammen; gebräuchlich ist auch die Benennung nach beiden Mathematikern. Auf die von Gottlob Frege unabhängig gefundenen Ergebnisse (1879/84) gehen wir hier aus Platzgründen nicht ein.

Dedekind konnte aus der mengentheoretischen Konstruktion seiner „Ketten“ die Ordnungsrelation auf den natürlichen Zahlen und das Beweisprinzip der vollständigen Induktion herleiten. In der rein axiomatischen Fassung wird letzteres zu einem der Axiome, während die Ordnungsrelation sich nicht in den Axiomen wiederfindet. Da Dedekinds Beweis des Rekursionssatzes aber die Ordnung benutzt, lässt er sich nicht einfach auf die axiomatische Fundierung hinübertransportieren. Diese Lücke 

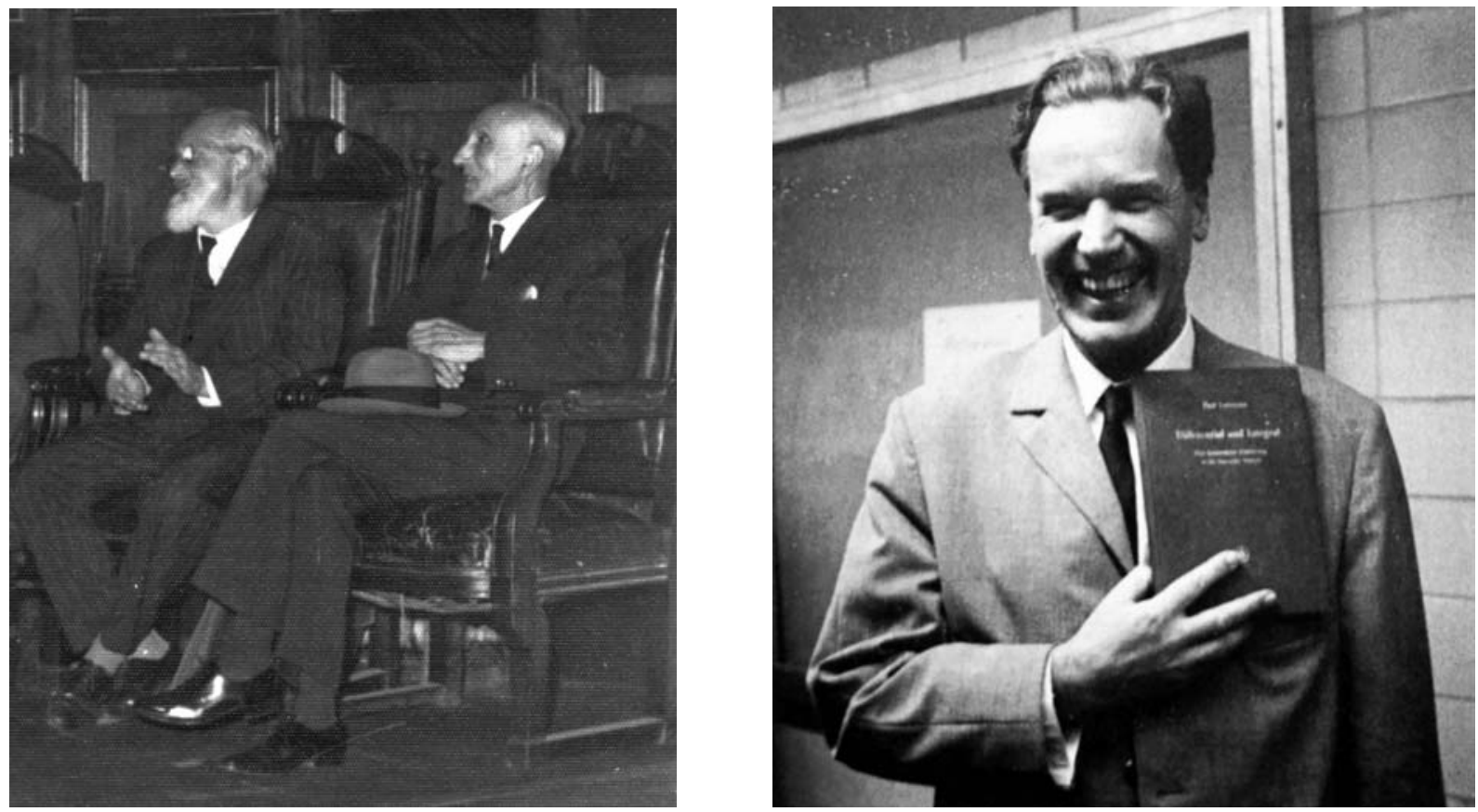

Abbildung 3. Links: Carlos Grandjot und Carlos Videla, ca. 1960. Rechts: Paul Lorenzen, 1971

wurde lange nicht beachtet und führte zu „Grandjots Einwand" im Sommer 1927. Grandjot ging in seiner Vorlesung pragmatisch mit dem Problem um: Er postulierte die Existenz der Addition und Multiplikation als weitere Axiome [13]. Es bleiben für den Zeitraum bis 1930 zwei Fragen. Erstens: Gab es Mathematiker, denen die Lücke vor 1927 aufgefallen war, und warum gehörte Landau nicht dazu? Zweitens: Welche Lösung fand Landau für sein Buch?

Nach Dedekinds Tod am 12.2.1916 hielt Landau im Mai 1917 eine Gedächtnisrede in der Göttinger Gesellschaft der Wissenschaften. Interessanterweise bat er für die Würdigung von „Was sind und was sollen die Zahlen?" Zermelo um Hilfe und verwendete dessen Analyse. Obwohl er beispielsweise im Wintersemester 1909/10 an Zermelos Vorlesung „Über die logischen Grundlagen der Mathematik" in Göttingen teilgenommen hatte, ${ }^{9}$ war Landau wohl mit seinem eigentlichen Arbeitsgebiet, der analytischen Zahlentheorie, stets so ausschließlich beschäftigt, dass er sich in den Grundlagenthemen nicht genügend auskannte und ihm so die lückenhafte Herleitung nicht auffiel. Andererseits ist belegt, dass Erhard Schmidt ${ }^{10}$ und John von Neumann ${ }^{\prime \prime}$ die Lücke kannten. Auch bei Zermelo, Hermann Weyl und im Hilbertschen Umfeld, z. B. bei Paul Bernays und Hellmuth Kneser, dürfte das der Fall gewesen sein.

Wie man dem „Vorwort für den Kenner“ entnehmen kann, wandte sich Landau an von Neumann und sie arbeiteten einen Beweis aus, der aus den Axiomen die Ordnung gewinnen sollte, um dann wie bei Dedekind fortzufahren. Dieses Vorgehen passte aber vom Stil nicht zum übrigen Buch und nahm auch zu viel Platz in Anspruch.
Bernays erinnerte sich dann an eine Bemerkung von László Kalmár, der im Sommersemester 1929 als Gaststudent in Göttingen gewesen war. Die Einzelheiten finden sich in dem auf Seite 42 abgedruckten Ausschnitt eines Interviews mit Kalmár aus dem Jahr 1972 [23]. Die Lösung war ein speziell auf die Addition und Multiplikation zugeschnittener Existenzbeweis, den wir Landau-KalmárMethode nennen wollen, um ihn von Kalmárs Ergebnis von $1940 \mathrm{zu}$ unterscheiden, welches ein Thema des nächsten Abschnitts sein wird.

\section{Geschichte des Dedekindschen Rekursionssatzes ab 1930}

Der erste Band der bereits erwähnten „Modernen Algebra" van der Waerdens erschien ebenfalls 1930, kurz nach Landaus „Grundlagen der Analysis“, sodass van der Waerden bei der Definition der Addition und Multiplikation natürlicher Zahlen auf Landau verweisen konnte. Nachdem er wie Landau die Ordnung eingeführt hat (,Ist $a=b+u$, so schreibt man $a>b$, oder auch $b<a$."), beweist er den Rekursionssatz. ${ }^{12}$ Aufgrund der Popularität der Bücher Landaus und van der Waerdens blieb dieses Vorgehen lange der Standard, und zwar bis ungefähr 1953. ${ }^{13}$

Bereits in den Jahren 1939/40 waren neue Beweise des Rekursionssatzes von Paul Lorenzen [29] und László Kalmár [22] erschienen, in denen ohne vorherige Einführung der Ordnung der Rekursionssatz direkt aus den Axiomen hergeleitet wird (siehe auch Abbildung 4). Alle rekursiv definierten Begriffe können damit in einheitlicher 
Weise begründet werden. ${ }^{14}$ Kurz gesagt besteht Kalmárs Methode aus der Konstruktion der gesuchten Funktion aus ihren „Keimen“ (,von unten“), während sie bei Lorenzen mit Hilfe des Durchschnitts aus allen die Rekursionsbedingung erfüllenden Relationen gebildet wird, d.h. „von oben“. Diese Methoden setzten sich ab 1954 durch und wir beschreiben im Weiteren verschiedene Phasen im Umgang mit dem Thema bis 1990.

\section{Lehrerausbildung}

Ein wichtiger Impuls in den 1950er und 1960er Jahren war die Wiederbegründung der Internationalen Mathematischen Unterrichtskommission (IMUK) im Jahr $1952^{15}$ und die damit zusammenhängenden Anstrengungen zur Verbesserung der Lehrerausbildung.

In der Vorlesung „Wissenschaftliche Grundlagen der Schulmathematik" [24] behandelte Hellmuth Kneser im Sommersemester 1954 in Tübingen als erstes Thema den Rekursionssatz basierend auf Kalmárs Artikel von 1940. Dann erschien 1958 der erste Band der von Behnke et al. herausgegebenen IMUK-Bände „Grundzüge der Mathematik“, für die die Planung sechs Jahre vorher in Oberwolfach begonnen hatte. Dort wird von Günter Pickert und Lilly Görke der Rekursionssatz mit der Methode von Lorenzen dargestellt [34].

Für unser Thema war die Zeit von 1957 bis 1968 sehr fruchtbar. Von Hao Wang erschien 1957 eine Analyse des Briefs von Dedekind an $\mathrm{H}$. Keferstein, der die Entstehungsgeschichte von „Was sind und was sollen die Zahlen?" beleuchtet [45]. Walter Felscher und Jürgen Schmidt nutzten in [I I] (1958) das Konzept der (transitiven) Hülle, um die Herleitung der Ordnungsrelation aus den Dedekind-Peano-Axiomen besonders übersichtlich darzustellen.

Die Definition durch vollständige Induktion.

$$
\text { Von P. Lorenzen in Göttingen. }
$$

Ph. Furtwängler zum 70. Geburtstag.

Unter Voraussetzung der Peanosehen Axiome der natürlichen Zahlen wird gewöhnlich die Addition definiert durch die Forderungen: $a+1=a^{\prime}, a+b^{\prime}=(a+b)^{\prime}$, wobei mit $c^{\prime}$ der ${ }_{n}$ Nachfolger ${ }^{a}$ von $c$ bezeichnet ist. $\mathrm{Da} b$ dadurch $a+b$ für alle $a$ und $b$ eindeutig definiert ist, wird als eine unmittelbare Folgerung aus dem Induktionsaxiom hingestellt. Wie Landau im Vorwort seines Buches „Grundlagen der Analysis“ aber auseinandersetzt, hat Grandjot darauf hingewiesen, dab dies nicht zulässig ist. Die Sehwierigkeit liegt darin, daß man zeigen mub, dab $a+b$ eindeutig definiert ist.

Dies steht alles schon bei Dedekind (Was sind und sollen Zahlen?). Dedekind führt nämlich vor der Addition die Ordnung der natirlichen Zahlen ein und beweist dann erst den "Satz von der Definition durch vollständige Induktion ${ }^{\star}$. Er bemerkt ausdrícklich, dab sich dieser Satz nicht in der gleichen Weise verallgemeinern labt wie das Induktionsaxiom (s. u.).

Landa u benutzt zur Definition von $a+b$ einen Kunstgriff, der von Kalmár stammt, $\sum_{v=1}^{n} a_{v}$ wird erst nach Einfuhrung der Ordnung definiert, da die Peanosche Definition

$$
\sum_{v=1}^{1} a_{v}=a_{1}, \quad \sum_{v=1}^{n^{\prime}} a_{v}=\sum_{v=1}^{n} a_{v}+a_{n^{\prime}}
$$

ebenfalls nicht ohne weiteres stichhaltig ist.

Abbildung 4. Beginn des Artikels von Paul Lorenzen, 1939

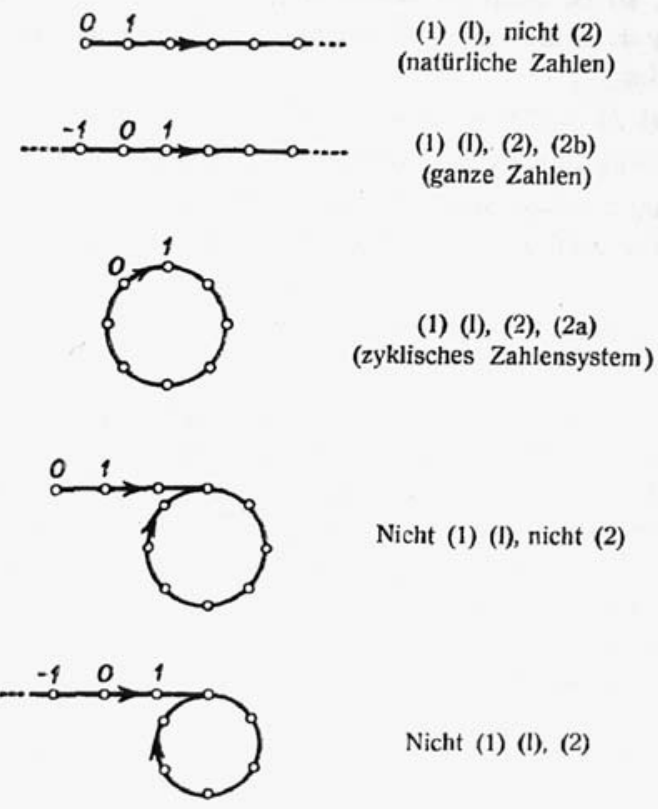

Fig. 1. Systeme, in denen das Induktionsaxiom (1) (II) gilt

Abbildung 5. Eine Abbildung aus dem Artikel [27] von Hanfried Lenz

Hanfried Lenz untersuchte 1958 in [27] Strukturen, die abgeschwächten Axiomensystemen genügen. Verzichtet man beispielsweise auf die Injektivität der Nachfolgerabbildung, behält aber die Forderung, dass das ausgezeichnete Element (hier die 0) nicht in ihrem Bild liegt, und das Induktionsaxiom bei, dann erfüllt dies die vierte Familie in Abbildung 5. Eine ähnliche Studie wurde im gleichen Jahr von Leon Henkin durchgeführt [19] und erschien 1960 im American Mathematical Monthly. ${ }^{16}$ Interessant ist hier die Unterscheidung von Peano- und InduktionsModellen. Bei letzteren verlangt man nur die Gültigkeit des Induktionsaxioms und, ähnlich zu den Ergebnissen von Lenz, gibt es dann außer den natürlichen Zahlen zusätzliche Strukturen, die den Rekursionssatz nicht erfüllen, für die man aber dennoch die Addition und Multiplikation mit der Landau-Kalmár-Methode definieren kann. ${ }^{17}$

Dieses Vorgehen leistet meines Erachtens auch einen didaktischen Beitrag: Durch die Analyse der zusätzlichen Strukturen werden die Beweisverfahren verständlicher. Man kann zum Beispiel die für den Beweis „,von unten“ benötigte Existenz von Anfangsstücken bei der erwähnten zyklischen Struktur $\mathbb{Z} / 7 \cdot \mathbb{Z}$ untersuchen (ein Anfangsstück ist eine Teilmenge der betrachteten Struktur mit der Eigenschaft, dass sie mit jedem Element der Form $n^{\prime}$ auch das „Vorgänger“-Element $n$ enthält) und findet, dass es dort keine solche echte Teilmenge gibt. Durch die Betrachtung mehrerer Beispiele wird deutlich, dass tatsächlich alle Axiome in den Beweisen des Rekursionssatzes benötigt werden.

In den Zeitabschnitt bis 1968 fallen außerdem die „Naive set theory" [18] von Paul Halmos und die Bücher 
Interview mit László Kalmár

Könnten wir etwas über Ihren Studienaufenthalt in Göttingen hören?

Kalmár: Göttingen war damals das Mekka der Mathematiker, in erster Linie wegen David Hilbert, er war ein Universalgenie und ging der Reihe nach durch alle Gebiete der Mathematik, warf überall die aktuellsten Probleme auf und löste sie. Außer Hilbert zogen noch Landau, Courant und Herglotz die Mathematiker der Welt nach Göttingen. Und es gab dort junge, begabte Privatdozenten, von denen man ebenfalls eine Menge lernen konnte. [...]

Ich ging nach Göttingen, als das Semester in Szeged schon zu Ende war und die Sommerferien begonnen hatten. In Göttingen hingegen war das Sommersemester [1929] noch in vollem Gange. Natürlich versuchte ich, mit Hilbert in Kontakt zu kommen. Er war schon alt, und es kam vor, dass ihm in seinen Vorlesungen über die Mengentheorie die Kreide aus der Hand fiel. ${ }^{18} \mathrm{Er}$ hatte einen sehr guten Privatdozenten, Bernays, der half Hilbert, sich hinzusetzen, nahm die Kreide und setzte die Vorlesung fort. Inzwischen döste Hilbert ein paar Minuten, dann sah er auf, hörte kurz zu, was Bernays sagte, nahm dann die Kreide wieder in die Hand und setzte seine Vorlesung fort. Bei einer Gelegenheit konnte ich durch die Vermittlung von Bernays sogar mit Hilbert sprechen.

Auch mit Landau wollte ich mich sehr gern treffen. Schon seit der Kindheit hatten mich die Primzahlen besonders interessiert, ich wollte alles über sie wissen. Von Professor Dávid hatte ich damals Landaus zweibändige Monografie über die Primzahlen geliehen bekommen, später lernte ich auch sein dreibändiges Werk über die Zahlentheorie kennen. Aber in Göttingen sagte man mir, Landau empfange niemanden, weil er an seinem neuen Buch arbeite. Ich meldete mich für Landaus funktionentheoretisches Seminar an. Er war bereit, mich aufzunehmen und gab mir sogar ein Thema für einen Vortrag. Auch nach dem Seminar konnte man nicht mit ihm sprechen, er verschwand immer augenblicklich. Er teilte seine Zeit gut ein!

Als ich wieder in Szeged war, bekam ich allerdings von Fenchel, einem von Landaus Assistenten, eine wortkarge Karte. Demnach hatte Landau von Bernays über meine Anmerkung im Zusammenhang mit den Grundlagen der Arithmetik, mit den Peano-Axiomen gehört, und habe nun folgende Fragen: $\mathrm{Ob}$ ich diese meine Anmerkungen publiziert hätte? Wenn ja, solle ich ihm die Publikationsdaten mitteilen. Wenn nicht, dann fragte er, ob ich gestatte, dass er sie in seinem Buch „Grundlagen der Analysis“, an dem er gerade arbeite, publizierte.

Ich musste eine gute Stunde nachdenken, auf welche Anmerkung von mir sich Landaus Fragen bezogen. Endlich fiel mir ein, dass Hilbert in einer Vorlesung die Addition und Multiplikation der sogenannten transfiniten Zahlen, einer ins Unendliche gehenden Verallgemeinerung der natürlichen Zahlen, abweichend von der üblichen mengentheoretischen Behandlung auf die Weise eingeführt hatte, wie man in der axiomatischen Arithmetik üblicherweise diese Operationen

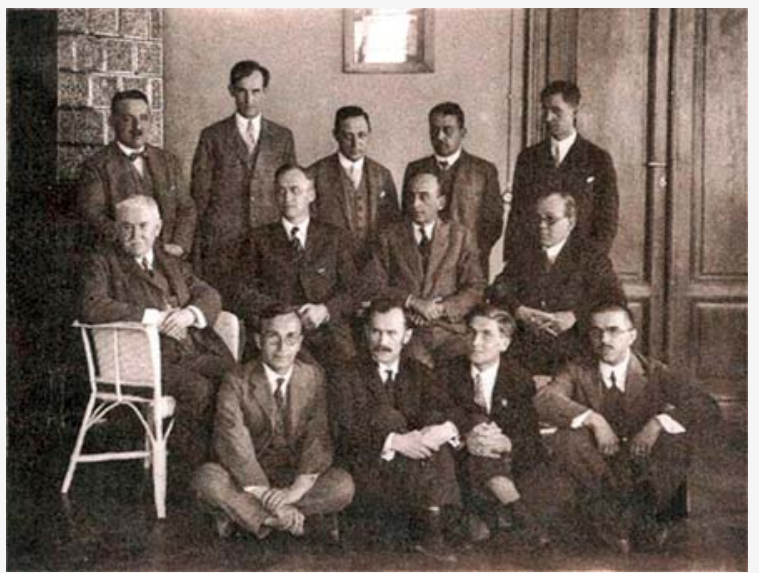

Abbildung 6. Konferenzfoto Szeged, 1928. I. Reihe: Tibor Radó, István Lipka, László Kalmár, Pál Szász. 2. Reihe: József Kürschák, George D. Birkhoff, O.D. Kellogg, Leopold Fejér. 3. Reihe: Frigyes Riesz, Béla Kerékjártó, Alfréd Haar, Julius König, Rudolf Ortvay.

für die natürlichen Zahlen einführt. Die These, die zeigt, dass die Definition mit der sogenannten transfiniten Rekursion berechtigt ist, bewies er meiner Ansicht nach komplizierter als nötig. Deshalb fragte ich Bernays nach der Vorlesung, warum Hilbert es nicht so gemacht habe, wie es in der Arithmetik üblich war. Bernays fragte daraufhin, was ich unter ,wie es in der Arithmetik üblich ist" verstehe. Beim Abendessen sagte ich ihm, dass ich mir für mich überlegt hatte, wie man auf der Grundlage des Axiomensystems aus John von Neumanns Artikel „Zur Hilbertschen Beweistheorie“ - von dem Neumann behauptet, es sei ausreichend für den Aufbau der Arithmetik - die Berechtigung der Definition der Addition und Multiplikation durch Rekursion beweisen kann. Ich dachte damals, das stellen sich alle so vor.

Landau interessierte diese meine Bemerkung, die ich für unwesentlich hielt, weil ihm, wie aus seinem später erschienenen Buch „Grundlagen der Analysis“ hervorging, zuvor folgender Fall - das ,schreckliche Abenteuer“, wie er im Vorwort zu seinem Buch schrieb - zugestoßen war. Er hatte eine Vorlesung über den axiomatischen Aufbau der Arithmetik nach den Peano-Axiomen gehalten. Dabei hatte er die Berechtigung ihrer Definition durch Rekursion falsch bewiesen. Darauf hatte ihn sein Assistent Grandjot aufmerksam gemacht. Für Landau bedeutete das einen derartigen Schock - er hielt sich bekanntlich für ein Muster an Präzision -, dass er ein Buch schreiben musste. [...]

Eigentlich begann damit mein Kontakt mit der mathematischen Logik: Nach diesem Fall hatte ich den Eindruck, es lohne sich, wenn ich mich damit beschäftigte. Außerdem interessierte mich Hilberts Programm der Beweistheorie, das ich in Neumanns oben genanntem Artikel kennengelernt hatte. In Budapest hatten wir nämlich nichts über mathematische Logik gehört, höchstens vielleicht einige spöttische Bemerkungen, dass Hilbert wieder etwas geschrieben habe, was nur die „Schriftgelehrten“ verstehen. Gewiss, Fejér erwähnte achtungsvoll die mathematische Logik und die Mengentheorie, aber er selbst beschäftigte sich nicht damit. ${ }^{19}$ 
[28] von Lenz und [7] von Solomon Feferman, die alle den Rekursionssatz nach Lorenzen beweisen. Jürgen Schmidt verbindet in [37] (1966) eine Einführung in die Mengenlehre mit historischen Anmerkungen und beweist den Rekursionssatz ebenfalls nach Lorenzen. Den Beweis über Anfangsstücke nach Kalmár wählt Arnold Oberschelp in seinem Buch aus dem Jahr 1968 [32]. In der Zeitschrift „Der Mathematikunterricht“ erschien 1967 ein Heft mit dem Schwerpunktthema „Die natürlichen Zahlen" mit mehreren Beiträgen zu Beweis und Definition durch Induktion/Rekursion. ${ }^{20}$

Für die Folgezeit bis 1990 ist der große Erfolg des Buchs „Zahlen“ zu erwähnen [3I], in dem Klaus Mainzer das Thema gut verständlich darstellt. In der deutschsprachigen Literatur $^{21}$ gibt es außerdem herausragende Beiträge von Walter Felscher [10], Heinz Lüneburg [30] und Konrad Jacobs [2I]. Felscher stellt ausführlich die mengentheoretischen Grundlagen dar, geht auf die Geschichte ein und zieht Querverbindungen zu verwandten algebraischen Strukturen wie freien Monoiden und unären Algebren. Lüneburg geht in knappem Stil vor und gibt auf wenigen Seiten einen variantenreichen Überblick. Sein Buch ist dem 100-jährigen Jubiläum des Erscheinens von „Was sind und was sollen die Zahlen?"“ gewidmet. Jacobs' Buch ist aus Vorlesungen mit dem Titel ,Mathematik für Philosophen" hervorgegangen und didaktisch sehr gut gelungen. $^{22}$

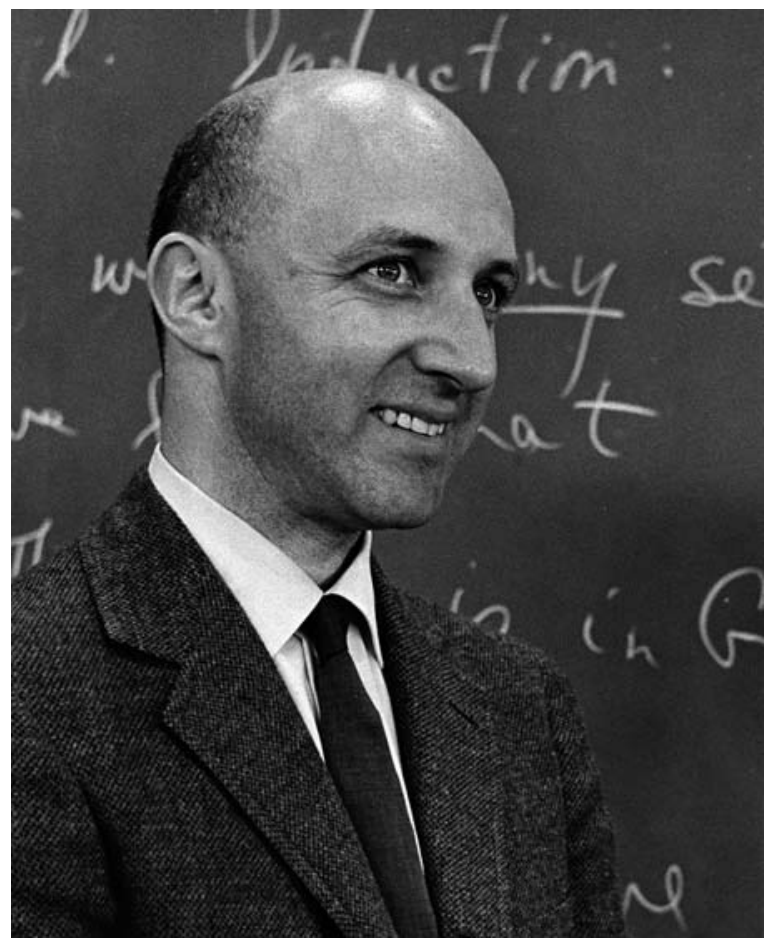

Abbildung 7. „Induktion - Der Film“. Leon Henkin bei den Dreharbeiten des Films ,Mathematical Induction“, 1960

\section{Abschließende Bemerkungen}

Wenn der Leser oder die Leserin nun selbst Beweise des Rekursionssatzes studieren möchte, dann seien zu Beginn die Texte von Halmos [18], Henkin [19] und Mainzer [3I] empfohlen. Zur Vertiefung gibt es dann die im vorigen Abschnitt angegebenen Bücher von Jacobs, Lüneburg und Felscher und die übrige zitierte Literatur. Je nach Verwendungszweck lässt sich so die passende Darstellungsform finden.

Dieser Artikel soll auch dazu dienen, vor der „Lücke“ zu warnen, denn auch heute kann es Mathematikern - so wie Landau 1927 - passieren, dass sie den Unterschied zwischen Beweis und Definition durch Induktion übersehen.

Recht unerforscht ist wohl der Status der beiden Bücher des niederländischen Mathematikers Fred Schuh (Abbildung 8 und [38]). Diese wurden von Hans Hermes in [20] angegeben und enthalten eine frühere Darstellung der Grundlagen der Analysis als die von Landau. Schließlich soll anhand einer aktuellen Veröffentlichung von Sergei Ovchinnikov [33] erwähnt werden, dass die LandauKalmár-Methode heute wieder verwendet und dort mit den späteren Beweisvarianten kombiniert wird. Zu verwandten Themen lese man auch kürzlich erschienene Artikel in den Mitteilungen und im Jahresbericht der DMV, so z. B. das Interview mit Günter Pickert [4] und Das Induktions-Prinzip von Ulrich Felgner [9] sowie natürlich „Carlos Grandjot. Drei Jahrzehnte der Mathematik in Chile: 1930-1960“ [16], den Ausgangspunkt unserer Untersuchung.

\section{Danksagungen}

Mein Dank gilt Karin Erxmeyer (Bad Arolsen) und Christina Kunze (Berlin) für die Übersetzungen aus dem Spanischen und Ungarischen, sowie Ulrich Hunger (Göttingen), Claudio Gutiérrez (Santiago) und Gerhard Wagenitz (Göttingen). Für Abbildungs- und Zitiererlaubnisse danke ich dem Universitätsarchiv Göttingen, dem Dolph Briscoe Center for American History für Abb. 7, der AMS für die Verwendung des Photos von Paul Lorenzen in Abbildung 3. Es stammt aus dem Buch I Have a Photographic Memory von Paul Halmos, AMS, 1987. Das Konferenzphoto Szeged findet man in commons.wikimedia.org und das Behnke-Zitat im Archiv des Mathematischen Forschungsinstituts Oberwolfach.

Anmerkungen

I. Siehe z. B. Birgit Bergmann/Moritz Epple: ,Jüdische Mathematiker in der deutschsprachigen akademischen Kultur", Springer, 2009, S. $173 / 174$.

2. Dieses und das folgende Zitat stammen aus der Personalakte Karl Grandjots [1].

3. Zum Vergleich kann die Entstehung von Courant/Robbins: „What is Mathematics?“', 194I, herangezogen werden. Siehe hierzu 

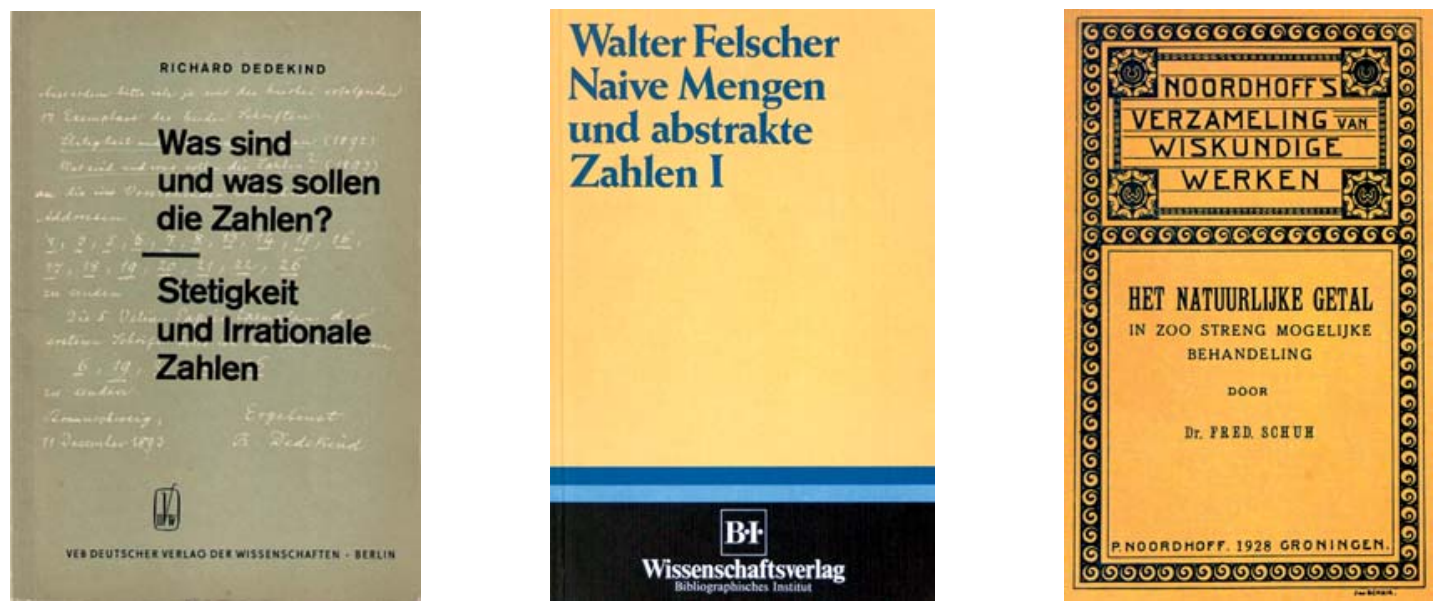

Abbildung 8. V. I. n. r.: Dedekinds „Zahlen“-Schriften in einer Ausgabe aus dem Jahr 1965 (mit einem Vorwort von Günter Asser [1926-2015]), Walter Felschers Buch, 1978, und Fred Schuhs Buch, 1928 [39]

Constance Reid: „Courant in Göttingen and New York“, Springer, 1976.

4. Siehe Siegmund-Schultze [40], S. 99, 122, 291.

5. In der Einleitung der „Algebra Abstracta“ schreibt Grandjot über Emmy Noether, deren Schüler und Kollege er von 1919 bis 1929 war und deren Vorlesungen eine der Grundlagen der „Modernen Algebra“ waren: „Und ich möchte nicht vergessen, die herausragendste weibliche Person der Geschichte der Mathematik zu erwähnen, die berühmte Emmy Noether. Ihr verdanken wir einen großen Teil des Fortschritts im 'abstrakten Denken' in der modernen Mathematik. Alle heutigen Forscher auf dem Gebiet der abstrakten Algebra sind entweder direkte oder indirekte Schüler Emmy Noethers." (Übersetzung: Karin Erxmeyer). Siehe auch [25]

6. Siehe [16], S. II.

7. Nach Hermann Weyl: „Philosophie der Mathematik und Naturwissenschaft“, Oldenbourg, 1927 (Abschnitt 6, „Die natürlichen Zahlen").

8. Siehe zum Beispiel [6], S. 84

9. Siehe [6], S. II5, 282.

10. Erhard Schmidts Assistent Georg Feigl hatte in seiner Besprechung von Landaus "Grundlagen der Analysis" im Jahrbuch über die Fortschritte der Mathematik (Band 56) darauf hingewiesen, dass Schmidt schon länger „Bedenken gegen die übliche induktive Definition der Addition" gehabt hatte. Erhard Schmidt, ein Schüler David Hilberts, hatte ein eigenes Axiomensystem für die natürlichen Zahlen entwickelt, siehe [36], welches von Feigl in Einführungsveranstaltungen in Berlin von 1920 bis 1934 verwendet wurde, siehe [8].

II. Bei von Neumann, einem Mitbegründer der axiomatischen Mengenlehre, ist dies nicht weiter erstaunlich. Als Beleg dient hier eine Anmerkung von Abraham Fraenkel in [12], S. 250. Von Neumann kannte auch Erhard Schmidt aus seiner Studienzeit in Berlin von 1921 bis 1923.

12. Dieser trägt hier noch die ältere Bezeichnung Existenz- und Eindeutigkeitssatz über die „Definition (oder Konstruktion) durch vollständige Induktion“. Zur Entstehungsgeschichte siehe [43].

13. Siehe hierfür die folgende Literatur: Feigl/Rohrbach [8, S. 270283]; P. Bernays [3, S. 197]; F. Bachmann [2, S. 4]; I. W. Proskurjakow [35, S. I I4-128]; A. Vogel [4I, S. 10-15] und H. Hermes [20, S. 1921, 70-72].

14. Der Beitrag Kalmárs zu Landaus Buch betraf nur die Einführung der Addition und der Multiplikation, während er 1940 den Rekursionssatz bewies. Dies wird gelegentlich verwechselt, z. B. in $[28, \mathrm{~S}$. 37].

15. Hierzu das Gästebuch des Forschungsinstituts Oberwolfach, 23./24. 8. 1952: „Beratungen zur Neugründung der IMUK und ihres deutschen Unterausschusses. Wir wissen noch nicht, welche Pläne reifen werden. Wir haben uns aber sehr gut verstanden, da/obwohl dieses Mal die Mathematik schon beim Kindergarten anfing." (Heinrich Behnke)

16. Die Mathematical Association of America drehte 1960 mit Leon Henkin sogar einen Film über die Induktion, siehe Abbildung 7. Henkin war auch Koautor des Buchs „Retracing elementary mathematics" (1962), welches den Aufbau des Zahlensystems ähnlich streng wie Landau durchführt, aber mehr Erläuterungen enthält.

17. Henkins Artikel [19] enthält im letzten Abschnitt eine auf Alonzo Church zurückgehende Information in Bezug auf den Beweis von Lorenzen. Es wird dort behauptet, dass der Beweis unabhängig auch von Hilbert/Bernays (Grundlagen der Mathematik, Band 2, Springer 1939) gefunden wurde, was aber aufgrund eines Kommentars von Bernays in der zweiten Auflage (1970, S. 508/509) widerlegt ist.

18. Hilbert litt seit einigen Jahren an einer durch Vitamin $B_{12}$-Mangel verursachten Blutarmut (perniziöse Anämie).

19. Bei Lipót Fejér promovierten 1926 Kalmár und von Neumann (und 1934 Paul Erdős). Kalmár widmete seinen Artikel [22] Fejér zum 60. Geburtstag.

20. Autoren der Beiträge waren Hans Hermes, Arnold Kirsch, Paul Sengenhorst, Günter Pickert und Hans-Georg Steiner.

21. Dazu zählen wir hier auch das Buch [30] von Heinz Lüneburg.

22. Man beachte auch Band I, Kapitel III, §7 (Induktive Konstruktion), wo auf Band 2 verwiesen wird.

\section{Literatur}

[I] Universitätsarchiv Göttingen, Personalakte Karl Grandjot

[2] Bachmann, Friedrich: Aufbau des Zahlensystems, Encyklopädie der Mathematischen Wissenschaften, Neuauflage, Band I (I. Teil), Teubner, 1939

[3] Bernays, Paul: Hilberts Untersuchungen über die Grundlagen der Arithmetik, in David Hilbert: Gesammelte Abhandlungen, 3. Band, Springer, 1935

[4] Beutelspacher, Albrecht und Törner, Günter: Interview mit Professor Dr. Günter Pickert, Mitteilungen der DMV 23 (2015), 4858

[5] Dedekind, Richard: Gesammelte mathematische Werke, Band 3, Vieweg, 1932

[6] Ebbinghaus, Heinz-Dieter: Ernst Zermelo. An Approach to His Life and Work, Springer, 2007

[7] Feferman, Solomon: The number systems. Foundations of algebra and analysis, Addison-Wesley, 1964 
[8] Feigl, Georg und Rohrbach, Hans: Einführung in die höhere Mathematik, Springer, 1953

[9] Felgner, Ulrich: Das Induktions-Prinzip, Jahresbericht der DMV II 4 (20I2), 23-45

[10] Felscher, Walter: Naive Mengen und abstrakte Zahlen, Band I, Bibliographisches Institut, 1978

[II] Felscher, Walter und Schmidt, Jürgen: Natürliche Zahlen, Ordnung, Nachfolge, Archiv für mathematische Logik und Grundlagenforschung 4 (1958), 8I-94

[12] Fraenkel, Abraham: Abstract set theory, North-Holland, 1953

[13] Grandjot, Karl: Vorlesungsskripte, Mitschriften von Hans Schwerdtfeger (Grundlagen der Analysis, 1927) und Vojtěch Jarník (Algebra II - Galoissche Theorie, 1927/28)

[14] Grandjot, Carlos: Algebra Abstracta, Apartado de la Revista Universitaria (Universidad Católica de Chile) 25 (1940), 1958

[15] Gutiérrez, Claudio und Gutiérrez, Flavio: Carlos Grandjot, Tres Décadas de Matemáticas en Chile: 1930-1960, Boletín de la Asociación Matemática Venezolana II (2004), 55-84

[16] Gutiérrez, Claudio und Gutiérrez, Flavio: Carlos Grandjot. Drei Jahrzehnte der Mathematik in Chile: 1930-1960, arXiv: I410.3036, deutsche Übersetzung von [15] von Karin Erxmeyer (2014)

[17] Gutiérrez, Claudio: persönliche Mitteilung, 19.06.2012

[18] Halmos, Paul: Naive set theory, van Nostrand, 1960 (deutsche Übersetzung 1968)

[19] Henkin, Leon: On Mathematical Induction, American Mathematical Monthly 67 (1960), 323-338

[20] Hermes, Hans: Grundlagen der Analysis, Skript, Münster, 1953

[2I] Jacobs, Konrad: Resultate. Band 2: Der Aufbau der Mathematik, Vieweg, 1990

[22] Kalmár, László: On the possibility of definition by recursion, Acta Sci. Math. Szeged 9 (1940), 227-232

[23] Kalmár, László: Interview 1972 (ung.), übersetzt von Christina Kunze, Original erhältlich unter www.inf.u-szeged.hu/projectdirs/kalmar/elete.php

[24] Kneser, Hellmuth: Aus einer Vorlesung über den mathematischen Schulstoff, Math.-Phys. Semesterberichte 5 (1956), 80-91 (sowie Gesammelte Abhandlungen, de Gruyter, 2005, S. 617-802, für die ursprüngliche Vorlesung)

[25] Koreuber, Mechthild: Emmy Noether, die Noether-Schule und die moderne Algebra, Springer, 2015

[26] Landau, Edmund: Grundlagen der Analysis, Akademische Verlagsgesellschaft, Leipzig, 1930 (weitere Auflagen 1963, 1970, 2004, englische Übersetzung 195I)

[27] Lenz, Hanfried: Zur Axiomatik der Zahlen, Acta Math. Hungar. 9 (1958), 33-44

[28] Lenz, Hanfried: Grundlagen der Elementarmathematik, Deutscher Verlag der Wissenschaften, 1961

[29] Lorenzen, Paul: Die Definition durch vollständige Induktion, Monatshefte für Math. und Physik 47 (1939), 356-358

[30] Lüneburg, Heinz: Tools and Fundamental Constructions of Combinatorial Mathematics, Bibliographisches Institut, 1989

[3I] Mainzer, Klaus: Natürliche, ganze und rationale Zahlen, in Ebbinghaus, Heinz-Dieter et al.: Zahlen, Springer, 1983 (mehrere Auflagen und Übersetzungen)

[32] Oberschelp, Arnold: Aufbau des Zahlensystems, Vandenhoeck \& Ruprecht, 1968

[33] Ovchinnikov, Sergei: Number Systems: An Introduction to Algebra and Analysis, AMS Publications, 2015

[34] Pickert, Günter und Görke, Lilly: Aufbau des Systems der reellen Zahlen, in H. Behnke et al.: Grundzüge der Mathematik, Band I, Vandenhoeck \& Ruprecht, 1958
[35] Proskurjakow, I. W.: Mengen, Gruppen, Ringe und Körper. Die theoretischen Grundlagen der Arithmetik, in Enzyklopädie der Elementarmathematik, Band I, Deutscher Verlag der Wissenschaften, Berlin, 1954 (russ. 195I)

[36] Rohrbach, Hans: Das Axiomensystem von Erhard Schmidt für die Menge der natürlichen Zahlen, Mathematische Nachrichten 4 (195I), 3I5-32I

[37] Schmidt, Jürgen: Mengenlehre, Bibliographisches Institut, 1966

[38] Schuh, Fred: Het getalbegrip in het bijzonder het onmeetbare getal, Noordhoff, 1927

[39] Schuh, Fred: Het natuurlijke getal in zoo streng mogelijke behandeling, Noordhoff, 1928 (bis Seite 60 erschienen als „Logische ontwikkeling van het begrip 'Natuurlijk getal'. I, II" in der Nieuw Tijdschrift Wiskunde 14 (1926/27), I75-190, 282-325)

[40] Siegmund-Schultze, Reinhard: Rockefeller and the Internationalization of Mathematics between the Two World Wars, Birkhäuser, 2001

[4I] Vogel, Alfred: Klassische Grundlagen der Analysis, Hirzel, 1952

[42] van der Waerden, Bartel: Moderne Algebra I, II, Springer, 1930/31

[43] van der Waerden, Bartel: On the sources of my book 'Moderne Algebra', Historia Mathematica 2 (1975), 3 I-40

[44] Wagenitz, Gerhard: Göttinger Biologen: 1737-1945. Eine biographisch-bibliographische Liste, Vandenhoeck \& Ruprecht, 1988

[45] Wang, Hao: The axiomatization of arithmetic, J. Symbolic Logic 22 (1957), 145-158

Dr. Christoph Lamm, Rückertstraße 3, 65I87 Wiesbaden christoph.lamm@web.de

Christoph Lamm (geb. 1969) studierte Mathematik in Göttingen, Reading/UK und Bonn. Promotion (Topologie) 1999 bei C.F. Bödigheimer und J. Przytycki. Er arbeitet als quantitativer Analyst im Bereich Counterparty Risk der Commerzbank. In den Mitteilungen der DMV erschien von ihm (zusammen mit Ekkehard Krätzel) 2013 ,Von Wiesbaden nach Tiflis. Die wechselvolle Lebensgeschichte des Zahlentheoretikers Arnold Walfisz", und er ist Herausgeber des 201 I bei der Paul Lazarus Stiftung in Wiesbaden erschienenen Buchs von Sophie GoetzelLeviathan (geb. Walfisz), „Der Krieg von Innen“. 